\title{
ON THE REGULARITY OF THE $2+1$ DIMENSIONAL EQUIVARIANT SKYRME MODEL
}

\author{
DAN-ANDREI GEBA AND DANIEL DA SILVA
}

(Communicated by James E. Colliander)

\begin{abstract}
One of the most interesting open problems concerning the Skyrme model of nuclear physics is the regularity of its solutions. In this article, we study $2+1$ dimensional equivariant Skyrme maps, for which we prove, using the method of multipliers, that the energy does not concentrate. This is one of the important steps towards a global regularity theory.
\end{abstract}

\section{INTRODUCTION}

The nonlinear $\sigma$-model of quantum field theory is given by the Lagrangian

$$
L_{\sigma}=-\frac{1}{2} S_{\mu}^{\mu}, \quad \text { where } \quad S_{\mu \nu}=g_{A B} \partial_{\mu} U^{A} \partial_{\nu} U^{B}
$$

is the pulled-back metric corresponding to $U: M \rightarrow N$, a map from a spacetime $\left(M, m_{\mu \nu}\right)$ to a complete Riemannian manifold $\left(N, g_{A B}\right)$. The critical points for this Lagrangian, also called harmonic maps or wave maps (depending on whether $m$ is a Riemannian or Lorentzian metric), have been a major research theme in the general area of partial differential equations for many years (see the excellent surveys [7] for harmonic maps and 21, 2], and [8] for wave maps).

For the purposes of this article, we restrict our discussion only to wave maps, with $(M, m)=\left(\mathbb{R}^{n+1}, \operatorname{diag}(-1,1, \ldots, 1)\right)$, which have a priori a conserved energy,

$$
E(U)=\frac{1}{2} \int_{\mathbb{R}^{n}}\left|U_{t}\right|_{g}^{2}+\left|\nabla_{x} U\right|_{g}^{2} d x .
$$

The case when $n=3$ and $N=\mathbb{S}^{3} \subset \mathbb{R}^{4}$ is referred to by the physicists as the classical nonlinear $\sigma$-model ([5], 4], 6]), describing the interactions between nucleons and pions. For finite energy wave maps, one can naturally associate to them a winding number

$$
Q=\frac{1}{2 \pi^{2}} \int_{\mathbb{R}^{3}} \operatorname{det}\left(U, \partial_{1} U, \partial_{2} U, \partial_{3} U\right)(x) d x .
$$

The constant in front of the integral is chosen such that $Q$ takes integer values. As the energy, this is also a conserved quantity (or topological charge in physical terminology), and it was Tony Skyrme's revolutionary idea which saw it as nothing but the baryon number of the nucleus, making it the first example of a topological soliton.

Received by the editors October 6, 2011.

2010 Mathematics Subject Classification. Primary 35L70, 81T13.

Key words and phrases. Skyrme model, global existence, nonconcentration of energy. 
From the mathematical point of view, one of the fundamental questions related to wave maps is their global regularity, of particular interest being the classical model and the one corresponding to $n=2$ and $N=\mathbb{S}^{2} \subset \mathbb{R}^{3}$. For the former, Shatah ([16]) showed that singularities form in finite time, even for smooth initial data, which is in accord with the physical intuition that, due to the attractive nature of the forces between pions, finite energy initial configurations of topological charge equal to one would shrink to a point, leading to a singularity formation. The $2+1$ dimensional case proved much more challenging, and the same result was obtained only recently (e.g., 9], [15, and [14]).

Another major contribution of Skyrme is his unifying model for mesons and baryons ([18, [19], 20]), which is a generalization of the classical model, with

$$
L_{S}=L_{\sigma}+\frac{\alpha^{2}}{4}\left(S^{\mu \nu} S_{\mu \nu}-S_{\mu}^{\mu} S_{\nu}^{\nu}\right)
$$

where $\alpha$ is a constant having the dimension of length. This theory is meant to fix the shortcomings of the classical model pointed out by Shatah; it has stable topological solitons, also called skyrmions. Therefore, the mathematical expectation with respect to the Euler-Lagrange equations associated to (4) is that finite energy initial data evolve into global smooth solutions (numerical evidence supporting this claim can be found in [1]). This is one of the most interesting open problems concerning the Skyrme model, which also includes questions concerning energy splitting and the asymptotic stability of the skyrmions. For more details, we refer the reader to [13] and the references therein.

The dynamics of the Skyrme model is described by a system of quasilinear wave equations, which is far more complex to study than the semilinear wave maps system. Perhaps this is one of the reasons why currently known results concerning its global regularity exist only for the equivariant case [11] and [3].

In this article, we take the first step towards proving global regularity for a Skyrme theory in $2+1$ dimensions (with $N=\mathbb{S}^{2} \subset \mathbb{R}^{3}$ ), which, in order to be Lorentz invariant and to have stable soliton-like solutions, needs an additional potential term in (4):

$$
\tilde{L}_{S}=L_{S}-V(U)
$$

The choices for $V$ vary in the literature, e.g., $V(U)=\lambda^{2}(1-n \cdot U)[10$ or $V(U)=$ $\lambda^{2}(1-n \cdot U)^{2}[12$, where $\lambda$ is a coupling constant having the inverse dimension of length and $n=(0,0,1)$ is the north pole of $\mathbb{S}^{2}$. However, the physical insight says that in what concerns the short-distance behavior (e.g., the development of singularities), the presence/absence of this new term is irrelevant, which is shown precisely later in Remark 2.7. This is why we decide to ignore the potential and work with the original Skyrme Lagrangian (4).

In line with the results for the $3+1$ dimensional problem, we choose to study equivariant critical maps of winding number 1 associated to $L_{S}$, i.e.,

$$
U(t, r, \omega)=(u(t, r), \omega), \quad u(t, 0)=0, \quad u(t, \infty)=\pi,
$$


where $u$ is the longitudinal angle and $(r, \omega)$ are the polar coordinates on $\mathbb{R}^{2}$. The Euler-Lagrange equations yield the following quasilinear wave equation for $u$ :

$$
\begin{aligned}
\left(1+\frac{\alpha^{2} \sin ^{2} u}{r^{2}}\right)\left(u_{t t}-u_{r r}\right) & -\left(1-\frac{\alpha^{2} \sin ^{2} u}{r^{2}}\right) \frac{u_{r}}{r} \\
& +\frac{\sin 2 u}{2 r^{2}}\left[\alpha^{2}\left(u_{t}^{2}-u_{r}^{2}\right)+1\right]=0 .
\end{aligned}
$$

A formal calculus shows that the conserved energy norm is given by

$$
\mathcal{E}[u](t)=\int_{0}^{\infty}\left[\left(1+\frac{\alpha^{2} \sin ^{2} u}{r^{2}}\right) \frac{u_{t}^{2}+u_{r}^{2}}{2}+\frac{\sin ^{2} u}{2 r^{2}}\right] r d r .
$$

We stress here that we are after a large data theory, for which the usual strategy is to combine a small data global well-posedness result with an argument that rules out energy concentration. In this article we achieve the latter, which is the more involved and nonstandard part of the proof, while the well-posedness component will be addressed in a forthcoming paper.

\section{Preliminaries}

Our main equation (6) is only at first glance quasilinear, as one can divide by the coefficient of the leading term, which is nondegenerate, and obtain a $1+1$ dimensional semilinear wave equation, for which the short-time existence of smooth solutions is, by now, standard. Moreover, from the radial symmetry, it is clear that if (6) develops a singularity, this has to appear at $r=0$.

Therefore, as the equation is reversible in time and it is invariant under time translations, we can assume, without any loss of generality, that our solution starts at time $t=T_{0}>0$ and the origin $(t, r)=(0,0)$ is the first possible blow-up point. Using a finite speed of propagation argument, we can thus prescribe initial data at $t=T_{0}$ and $r \leq T_{0}$ and solve (6) backward in time in

$$
\Omega=\left\{(t, r) \mid 0<t \leq T_{0}, r \leq t\right\} .
$$

The local theory allows us also to assume that $u$ is smooth in $\Omega$. Under these conditions, our main result can be formulated as follows:

Theorem 2.1. If $u$ is a smooth solution of (6) in $\Omega$, then its energy does not concentrate at the origin, i.e.,

$$
\lim _{T \rightarrow 0+} \int_{0}^{T}\left[\left(1+\frac{\alpha^{2} \sin ^{2} u}{r^{2}}\right) \frac{u_{t}^{2}+u_{r}^{2}}{2}+\frac{\sin ^{2} u}{2 r^{2}}\right] r d r=0 .
$$

Our energy analysis is mainly done on forward truncated cones, their mantels, and their spacelike sections, denoted by

$$
\begin{aligned}
K\left(t_{1}, t_{2}\right) & :=\left\{(t, r) \mid t_{1} \leq t \leq t_{2}, 0 \leq r \leq t\right\}, \\
C\left(t_{1}, t_{2}\right) & :=\left\{(s, s) \mid t_{1} \leq s \leq t_{2}\right\}, \\
\Sigma\left(t_{1}\right) & :=\left\{\left(t_{1}, r\right) \mid 0 \leq r \leq t_{1}\right\},
\end{aligned}
$$

where $0<t_{1} \leq t_{2} \leq T_{0}$. For narrower sections, we use

$$
\Sigma_{\lambda}\left(t_{1}\right):=\left\{\left(t_{1}, r\right) \mid \lambda t_{1} \leq r \leq t_{1}\right\},
$$

with $0<\lambda<1$. 
Next, we introduce the notation

$$
w:=1+\frac{\alpha^{2} \sin ^{2} u}{r^{2}}, \quad e:=w \frac{u_{t}^{2}+u_{r}^{2}}{2}+\frac{\sin ^{2} u}{2 r^{2}}, \quad m:=w u_{t} u_{r},
$$

where $e$ and $m$ are also called the energy density, respectively the momentum density. We can record our first proposition.

Proposition 2.2. Classical solutions of (6) satisfy the following two differential identities:

$$
\begin{gathered}
\partial_{t}\left(r\left[a e+b m+c w h(u) u_{t}\right]\right)-\partial_{r}\left(r\left[a m+b\left(e-\frac{\sin ^{2} u}{r^{2}}\right)+c w h(u) u_{r}\right]\right) \\
=r\left\{(A+B) \frac{u_{t}^{2}}{2}+(A-B) \frac{u_{r}^{2}}{2}+\left(b_{t}-a_{r}\right) m+\left(a_{t}+b_{r}-\frac{b}{r}\right) \frac{\sin ^{2} u}{2 r^{2}}\right. \\
\left.-c \frac{h(u) \sin 2 u}{2 r^{2}}+w h(u)\left(c_{t} u_{t}-c_{r} u_{r}\right)\right\},
\end{gathered}
$$

where

$$
\begin{gathered}
a=a(t, r), \quad b=b(t, r), \quad c=c(t, r), \quad h=h(u), \\
A:=w\left(a_{t}-b_{r}\right), \quad B:=-\left(1-\frac{\alpha^{2} \sin ^{2} u}{r^{2}}\right) \frac{b}{r}+c\left(2 w h^{\prime}(u)+\frac{\alpha^{2} h(u) \sin 2 u}{r^{2}}\right),
\end{gathered}
$$

and

$$
\begin{aligned}
& \partial_{t}\left(r u u_{t}\right)-\partial_{r}\left(r u u_{r}\right) \\
= & r\left\{\left[1-\frac{\frac{\alpha^{2} u \sin 2 u}{2 r^{2}}}{w}\right]\left(u_{t}^{2}-u_{r}^{2}\right)+\left[\frac{1-\frac{\alpha^{2} \sin ^{2} u}{r^{2}}}{w}-1\right] \frac{u u_{r}}{r}-\frac{\frac{u \sin 2 u}{2 r^{2}}}{w}\right\} .
\end{aligned}
$$

Proof. The first identity is obtained when we multiply our equation by $r\left(a u_{t}+\right.$ $\left.b u_{r}+c h(u)\right)$ and rearrange the terms conveniently, while the second one follows by rewriting $u_{t t}-u_{r r}$ using (6) directly.

For $(a, b, c)=(1,0,0)$, (9) is the energy differential identity

$$
\partial_{t}(r e)-\partial_{r}(r m)=0,
$$

which, integrated on $K\left(t_{1}, t_{2}\right) \subset \Omega$, yields

$$
E\left(t_{2}\right)-E\left(t_{1}\right)=F\left(t_{1}, t_{2}\right) .
$$

Here

$$
E(T)=\int_{\Sigma(T)} e \quad \text { and } \quad F(S, T)=\frac{1}{\sqrt{2}} \int_{C(S, T)} e+m
$$

are the energy of the time slice $t=T$, respectively the flux between the time slices $t=S$ and $t=T$ for the function $u 1$ Both of these quantities are positive definite, as obviously $e \geq 0$, while

$$
e+m=w \frac{\left(u_{t}+u_{r}\right)^{2}}{2}+\frac{\sin ^{2} u}{2 r^{2}} \geq 0 .
$$

We thus obtain:

\footnotetext{
${ }^{1}$ This is the motivation for the terminology associated with $e$ and $m$.
} 
Proposition 2.3. The energy is monotone and the flux decays to 0, i.e.,

$$
\begin{gathered}
E\left(t_{1}\right) \leq E\left(t_{2}\right) \quad \text { for } 0<t_{1} \leq t_{2} \leq T_{0}, \\
\lim _{T \rightarrow 0+} F(0, T)=0 .
\end{gathered}
$$

Remark 2.4. It follows immediately from (12) that

$$
0 \leq \lim _{T \rightarrow 0+} E(T)=l<\infty,
$$

and so, in order to prove the nonconcentration of energy, it suffices to demonstrate that $l=0$.

We now have all the ingredients to show that:

Proposition 2.5. A smooth solution $u$ for (6) in $\Omega$ is continuous at $(0,0)$ and

$$
|u(t, r)| \leq C\left(E\left(T_{0}\right)\right) r^{1 / 2}, \quad(\forall)(t, r) \in \Omega, t \ll 1 .
$$

Proof. For the functional

$$
I(z)=\int_{0}^{z}|\sin w| d w
$$

one has

$$
I(0)=0, \quad|I(z)|>0(z \neq 0), \quad \lim _{|z| \rightarrow \infty}|I(z)|=\infty .
$$

Using the co-rotational hypothesis (i.e., $u(t, 0)=0$ ), we can write

$$
I(u(t, r))=\int_{0}^{r}|\sin u(t, s)| u_{r}(t, s) d s,
$$

which implies, based on (12), that

$$
\begin{aligned}
|I(u(t, r))| & \lesssim\left(\int_{0}^{r} s d s\right)^{\frac{1}{2}}\left(\int_{\Sigma(t)} \frac{\sin ^{2} u(t, s)}{s^{2}} u_{r}^{2}(t, s)\right)^{\frac{1}{2}} \\
& \lesssim r E(t)^{1 / 2} \lesssim r E\left(T_{0}\right)^{1 / 2} .
\end{aligned}
$$

This obviously shows that $u$ is continuous at the origin, i.e.,

$$
\lim _{(t, r) \in \Omega \rightarrow(0,0)} u(t, r)=0 .
$$

For $t$ sufficiently small, we can then argue that

$$
u^{2}(t, r) \lesssim \int_{0}^{r}|\sin u(t, s)| \cdot\left|u_{r}(t, s)\right| d s \lesssim r E\left(T_{0}\right)^{1 / 2},
$$

which proves (15).

As an easy consequence, we obtain nonconcentration for one of the terms in $e$ :

Corollary 2.6. For a smooth solution $u$ of (6) in $\Omega$, the following holds:

$$
\lim _{T \rightarrow 0+} \int_{\Sigma(T)} \frac{\sin ^{2} u}{2 r^{2}}=0 .
$$

Remark 2.7. The presence of the potential term in (4) would add to $e$ only bounded expressions, which obviously do not concentrate. For example, if $V(U)=$ $\lambda^{2}(1-n \cdot U)$, then

$$
\lim _{T \rightarrow 0+} \int_{\Sigma(T)} \lambda^{2}(1-\cos u)=0 .
$$


Remark 2.8. From (16), we see that once we have a sequence $\left(t_{n}\right)_{n} \rightarrow 0$ with

$$
\lim _{n \rightarrow \infty} \int_{\Sigma\left(t_{n}\right)} \frac{\sin ^{2} u}{r^{2}} u_{r}^{2}=0
$$

it follows that

$$
\left|u\left(t_{n}, r\right)\right| \leq C_{n} r^{1 / 2}, \quad(\forall)\left(t_{n}, r\right) \in \Sigma\left(t_{n}\right),
$$

with $C_{n} \rightarrow 0$ as $n \rightarrow \infty$.

Remark 2.9. For general, finite energy smooth solutions of (6), we deduce, using the same functional as above, that

$$
|I(u(t, r))| \lesssim\left(\int_{0}^{r} \frac{\sin ^{2} u(t, s)}{s} d s\right)^{\frac{1}{2}} \cdot\left(\int_{0}^{r} u_{r}^{2}(t, s) s d s\right)^{\frac{1}{2}} \lesssim \mathcal{E}[u](t) .
$$

This implies that $u$ is a priori bounded, as the energy norm (7) is conserved in time.

\section{Main ARgument}

We follow closely the blueprint for proving nonconcentration of energy, as it was used for equivariant wave maps in [17. First, we show that the energy does not concentrate near the light cone, which implies, jointly with (17), that the energy corresponding to the $u_{t}^{2}$ and $\frac{\sin ^{2} u}{r^{2}} u_{r}^{2}$ terms decays to 0 . This result is then used to obtain nonconcentration for the $u_{r}^{2}$ density. Finally, we rely on all of these previous facts to prove the similar result for the $\frac{\sin ^{2} u}{r^{2}} u_{t}^{2}$ part of the energy.

Our main tools in this section are the differential identities (9) and (10), which are integrated on forward truncated cones $K\left(t_{1}, t_{2}\right) \subset \Omega\left(0<t_{1} \leq t_{2} \leq T_{0}\right)$. The strategy is to show that we can allow first $t_{1} \rightarrow 0$, independently of $t_{2}$, in the resulting equation, followed subsequently by $t_{2} \rightarrow 0$ in the previously obtained limit.

We apply this approach first for (9) with $(a, b, c)=(t, 0,0)$, deducing

$$
\int_{K\left(t_{1}, t_{2}\right)} e=t_{2} E\left(t_{2}\right)-t_{1} E\left(t_{1}\right)-\frac{1}{\sqrt{2}} \int_{C\left(t_{1}, t_{2}\right)} t(e+m) .
$$

On the other hand, using (13) and (14), we obtain

$$
\lim _{t_{1} \rightarrow 0} \int_{K\left(0, t_{1}\right)} e+t_{1} E\left(t_{1}\right)+\int_{C\left(0, t_{1}\right)} t(e+m)=0,
$$

which, coupled with the previous equality and the positivity of the two integrands, yields

$$
\int_{K\left(0, t_{2}\right)} e=t_{2} E\left(t_{2}\right)-\frac{1}{\sqrt{2}} \int_{C\left(0, t_{2}\right)} t(e+m) .
$$

Finally, using (13) again, we infer

$$
\lim _{T \rightarrow 0+} \frac{1}{T} \int_{K(0, T)} e=\lim _{T \rightarrow 0+} E(T)=l,
$$

which provides us with an equivalent way of showing that $l=0$. 
3.1. Behavior near the cone. Next, our goal is to show nonconcentration of energy near the light cone, i.e.,

$$
\lim _{T \rightarrow 0+} \int_{\Sigma_{\lambda}(T)} e=0
$$

for any fixed $0<\lambda<1$. As the proof is quite similar to the one in [17], we sketch here only the main steps.

First, we prove that

$$
\begin{aligned}
\left|\partial_{\xi}(r(e+m))\right| & \lesssim((e+m)(e-m))^{1 / 2}, \\
\left|\partial_{\eta}(r(e-m))\right| & \lesssim((e+m)(e-m))^{1 / 2},
\end{aligned}
$$

where $\eta=t+r$ and $\xi=t-r$ are the classical null coordinates. For $(a, b, c)=(0,1,0)$, (9) can be rewritten as

$$
\partial_{t}(r m)-\partial_{r}(r e)=-\left(1-\frac{\alpha^{2} \sin ^{2} u}{r^{2}}\right) \frac{u_{t}^{2}-u_{r}^{2}}{2}+\frac{\sin ^{2} u}{2 r^{2}}-\frac{\sin 2 u \cdot u_{r}}{r}=D,
$$

which, coupled with (11), implies

$$
\partial_{\xi}(r(e+m))=-\partial_{\eta}(r(e-m))=\frac{D}{2} .
$$

Therefore, in order to obtain (23)-(24), it is enough to verify

$$
D^{2} \lesssim(e+m)(e-m)
$$

which follows from the straightforward bound

$$
D^{2} \lesssim\left(1-\frac{\alpha^{2} \sin ^{2} u}{r^{2}}\right)^{2}\left(u_{t}^{2}-u_{r}^{2}\right)^{2}+\frac{\sin ^{4} u}{r^{4}}+\frac{\sin ^{2} 2 u \cdot u_{r}^{2}}{r^{2}} \lesssim(e+m)(e-m) .
$$

Next, integrating (23)-(24) on regions situated in the domain

$$
K_{\lambda}(0, T)=\{(t, r) \mid 0<t \leq T, \lambda t \leq r \leq t\},
$$

we deduce that

$$
\lim _{(t, r) \in K_{\lambda}(0, T) \rightarrow(0,0)} r^{2}(e-m)=0,
$$

which further implies that

$$
\lim _{T \rightarrow 0+} \int_{\tilde{C}_{\lambda}(T)} e-m=0,
$$

where $\tilde{C}_{\lambda}(T)$ is the surface described by

$$
t+r=(1+\lambda) T, \quad \lambda t \leq r \leq t .
$$

Finally, an integration of (11) yields

$$
\int_{\Sigma_{\lambda}(T)} e=F\left(\frac{1+\lambda}{2} T, T\right)+\frac{1}{\sqrt{2}} \int_{\tilde{C}_{\lambda}(T)} e-m,
$$

which, based on (13) and (25), proves (22).

However, the crucial piece of information needed in our argument is

$$
\lim _{T \rightarrow 0+} \int_{\Sigma(T)} \frac{r}{T} e=0,
$$


which follows immediately from (22) and

$$
0 \leq \int_{\Sigma(T)} \frac{r}{T} e \leq \lambda E(T)+\int_{\Sigma_{\lambda}(T)} e,
$$

an estimate that holds for any fixed $0<\lambda<1$.

3.2. Nonconcentration of energy for the $u_{t}^{2}$ and $\frac{\sin ^{2} u}{r^{2}} u_{r}^{2}$ densities. We integrate (9) for $(a, b, c)=(0, r, 0)$ on the truncated cone $K\left(t_{1}, t_{2}\right)$ to infer that

$$
\begin{aligned}
\int_{\Sigma\left(t_{2}\right)} r m+ & \int_{K\left(t_{1}, t_{2}\right)} u_{t}^{2}+\frac{\alpha^{2} \sin ^{2} u}{r^{2}} u_{r}^{2}=\int_{\Sigma\left(t_{1}\right)} r m \\
& +\frac{1}{\sqrt{2}} \int_{C\left(t_{1}, t_{2}\right)} r\left[\left(1+\frac{\alpha^{2} \sin ^{2} u}{r^{2}}\right) \frac{\left(u_{t}+u_{r}\right)^{2}}{2}-\frac{\sin ^{2} u}{2 r^{2}}\right] .
\end{aligned}
$$

Reasoning as in the start of this section, we can take $t_{1} \rightarrow 0$ and then divide by $t_{2}$ to deduce

$$
\begin{aligned}
\frac{1}{t_{2}} \int_{\Sigma\left(t_{2}\right)} r m+\frac{1}{t_{2}} & \int_{K\left(0, t_{2}\right)} u_{t}^{2}+\frac{\alpha^{2} \sin ^{2} u}{r^{2}} u_{r}^{2} \\
& =\frac{1}{\sqrt{2} t_{2}} \int_{C\left(0, t_{2}\right)} r\left[\left(1+\frac{\alpha^{2} \sin ^{2} u}{r^{2}}\right) \frac{\left(u_{t}+u_{r}\right)^{2}}{2}-\frac{\sin ^{2} u}{2 r^{2}}\right] .
\end{aligned}
$$

Using (26) and the trivial bounds

$$
|m| \leq e, \quad\left|\left(1+\frac{\alpha^{2} \sin ^{2} u}{r^{2}}\right) \frac{\left(u_{t}+u_{r}\right)^{2}}{2}-\frac{\sin ^{2} u}{2 r^{2}}\right| \leq e+m,
$$

we conclude that

$$
\lim _{T \rightarrow 0+} \frac{1}{T} \int_{K(0, T)} u_{t}^{2}+\frac{\alpha^{2} \sin ^{2} u}{r^{2}} u_{r}^{2}=0 .
$$

3.3. Nonconcentration of energy for the $u_{r}^{2}$ density. This is the point in the argument where we rely on (10), which, integrated on $K\left(t_{1}, t_{2}\right)$, leads to

$$
\begin{aligned}
\int_{\Sigma\left(t_{2}\right)} u & u_{t}-\int_{\Sigma\left(t_{1}\right)} u u_{t}-\frac{1}{\sqrt{2}} \int_{C\left(t_{1}, t_{2}\right)} u\left(u_{t}+u_{r}\right) \\
= & \int_{K\left(t_{1}, t_{2}\right)}\left[1-\frac{\frac{\alpha^{2} u \sin 2 u}{2 r^{2}}}{w}\right]\left(u_{t}^{2}-u_{r}^{2}\right)+\left[\frac{1-\frac{\alpha^{2} \sin ^{2} u}{r^{2}}}{w}-1\right] \frac{u u_{r}}{r}-\frac{\frac{u \sin 2 u}{2 r^{2}}}{w} .
\end{aligned}
$$

Based on Proposition 2.5, if we choose $t_{2}$ sufficiently small, then $u$ is small and we can use in $K\left(0, t_{2}\right)$ both (15) and the uniform bound $|u| \lesssim|\sin u|$, which allows us morally to think of $u$ as $\sin u$ everywhere in the above equation.

We discuss first the integrals on the bases of the cone and on its mantle. Using the Cauchy-Schwarz inequality, for $t \leq t_{2}$, we obtain the following set of estimates:

$$
\begin{aligned}
\left|\int_{\Sigma(t)} u u_{t}\right| & \lesssim t^{2}\left(\int_{\Sigma(t)} \frac{\sin ^{2} u}{r^{2}} u_{t}^{2}\right)^{1 / 2} \lesssim t^{2} E(t)^{1 / 2} \\
\left|\int_{C(0, t)} u\left(u_{t}+u_{r}\right)\right| & \lesssim t^{2}\left(\int_{C(0, t)} \frac{\sin ^{2} u}{r^{2}}\left(u_{t}+u_{r}\right)^{2}\right)^{1 / 2} \lesssim t^{2} F(0, t)^{1 / 2}
\end{aligned}
$$


which imply

$$
\lim _{T \rightarrow 0+} \frac{1}{T} \int_{\Sigma(T)} u u_{t}=\lim _{T \rightarrow 0+} \frac{1}{T} \int_{C(0, T)} u\left(u_{t}+u_{r}\right)=0 .
$$

For the last two terms in the integral on the cone, we can argue as

$$
\begin{aligned}
\mid \int_{K(0, t)}\left[\frac{1-\frac{\alpha^{2} \sin ^{2} u}{r^{2}}}{w}-1\right] & \frac{u u_{r}}{r}-\frac{\frac{u \sin 2 u}{2 r^{2}}}{w}\left|\lesssim \int_{K(0, t)}\right| \frac{u u_{r}}{r} \mid+1 \\
& \lesssim t^{3 / 2}\left(\int_{K(0, t)} \frac{\sin ^{2} u}{r^{2}} u_{r}^{2}\right)^{1 / 2}+t^{3}
\end{aligned}
$$

from which we deduce

$$
\lim _{T \rightarrow 0+} \frac{1}{T} \int_{K(0, T)}\left[\frac{1-\frac{\alpha^{2} \sin ^{2} u}{r^{2}}}{w}-1\right] \frac{u u_{r}}{r}-\frac{\frac{u \sin 2 u}{2 r^{2}}}{w}=0 .
$$

Also,

$$
\left|\int_{K(0, t)}\left(1-\frac{\frac{\alpha^{2} u \sin 2 u}{2 r^{2}}}{w}\right) u_{t}^{2}+\frac{\frac{\alpha^{2} u \sin 2 u}{2 r^{2}}}{w} u_{r}^{2}\right| \lesssim \int_{K(0, t)} u_{t}^{2}+\frac{\alpha^{2} \sin ^{2} u}{r^{2}} u_{r}^{2}
$$

implies, based on (27), that

$$
\lim _{T \rightarrow 0+} \frac{1}{T} \int_{K(0, T)}\left(1-\frac{\frac{\alpha^{2} u \sin 2 u}{2 r^{2}}}{w}\right) u_{t}^{2}+\frac{\frac{\alpha^{2} u \sin 2 u}{2 r^{2}}}{w} u_{r}^{2}=0 .
$$

Thus, taking in (28) first $t_{1} \rightarrow 0$, then dividing the limiting result by $t_{2}$, and finally allowing $t_{2} \rightarrow 0$, we conclude, based on (29)-(31), that

$$
\lim _{T \rightarrow 0+} \frac{1}{T} \int_{K(0, T)} u_{r}^{2}=0
$$

3.4. Nonconcentration of energy for the $\frac{\sin ^{2} u}{r^{2}} u_{t}^{2}$ density. For this last term we use (9) with $(a, b, c)=(0,0,1)$ and $h(u)=\sin u$ to infer, after integrating on $K\left(t_{1}, t_{2}\right)$, that

$$
\begin{aligned}
\int_{\Sigma\left(t_{2}\right)} w \sin u u_{t} & +\int_{K\left(t_{1}, t_{2}\right)}\left(w \cos u+\frac{\alpha^{2} \sin u \sin 2 u}{2 r^{2}}\right)\left(u_{r}^{2}-u_{t}^{2}\right)+\frac{\sin u \sin 2 u}{2 r^{2}} \\
& =\int_{\Sigma\left(t_{1}\right)} w \sin u u_{t}+\frac{1}{\sqrt{2}} \int_{C\left(t_{1}, t_{2}\right)} w \sin u\left(u_{t}+u_{r}\right) .
\end{aligned}
$$

We will be working as above, with $t_{2}$ sufficiently small, such that we can use (15) and deduce for $t \leq t_{2}$ that

$$
\begin{gathered}
\left|\int_{K(0, t)}\left(w \cos u+\frac{\alpha^{2} \sin u \sin 2 u}{2 r^{2}}\right)\left(u_{r}^{2}-u_{t}^{2}\right)+\frac{\sin u \sin 2 u}{2 r^{2}}\right| \lesssim \int_{K(0, t)} e \\
\lesssim t E(t)
\end{gathered}
$$




$$
\left|\int_{\Sigma(t)} w \sin u u_{t}\right| \lesssim\left(\int_{\Sigma(t)} w \sin ^{2} u\right)^{1 / 2}\left(\int_{\Sigma(t)} w u_{t}^{2}\right)^{1 / 2} \lesssim t E(t)^{1 / 2}
$$

$$
\begin{aligned}
\left|\int_{C(0, t)} w \sin u\left(u_{t}+u_{r}\right)\right| & \lesssim\left(\int_{C(0, t)} w \sin ^{2} u\right)^{1 / 2}\left(\int_{C(0, t)} w\left(u_{t}+u_{r}\right)^{2}\right)^{1 / 2} \\
& \lesssim t F(0, t)^{1 / 2} .
\end{aligned}
$$

These estimates allow us to apply our general strategy (i.e., first $t_{1} \rightarrow 0$, then divide by $t_{2}$, followed by $t_{2} \rightarrow 0$ ) to obtain

$$
\begin{aligned}
\lim _{T \rightarrow 0+} \frac{1}{T} \int_{\Sigma(T)} w \sin u u_{t} \\
\quad+\frac{1}{T} \int_{K(0, T)}\left(w \cos u+\frac{\alpha^{2} \sin u \sin 2 u}{2 r^{2}}\right)\left(u_{r}^{2}-u_{t}^{2}\right)+\frac{\sin u \sin 2 u}{2 r^{2}}=0 .
\end{aligned}
$$

As mentioned previously, due to (15), $u$ is small close to the tip of the cone and one can then replace, without any loss of generality, $\cos u$ by 1 in the second integral. Furthermore, using (17), (27), and (32), we can strip down more terms from the above limit, which leads to

$$
\lim _{T \rightarrow 0+} \frac{1}{T} \int_{\Sigma(T)} w \sin u u_{t}-\frac{1}{T} \int_{K(0, T)} \frac{\alpha^{2} \sin ^{2} u}{r^{2}} u_{t}^{2}=0 .
$$

We see that (35) is not enough to carry the day, and this is why we have to do a finer analysis of the first term in (37). From (27) we obtain the existence of a sequence $\left(t_{n}\right)_{n} \rightarrow 0$ satisfying (18); hence we can use the finer bound (19) to infer that

$$
\int_{\Sigma\left(t_{n}\right)} w \sin ^{2} u \lesssim \int_{\Sigma\left(t_{n}\right)} C_{n}^{2} r+C_{n}^{4} \lesssim C_{n}^{2} t_{n}^{3}+C_{n}^{4} t_{n}^{2}
$$

which implies, based on (35), that

$$
\lim _{n \rightarrow \infty} \frac{1}{t_{n}} \int_{\Sigma\left(t_{n}\right)} w \sin u u_{t}=0 .
$$

Coupling this limit with (37), (21), (17), (27), and (32), we conclude that

$$
\lim _{T \rightarrow 0+} \frac{1}{T} \int_{K(0, T)} \frac{\alpha^{2} \sin ^{2} u}{r^{2}} u_{t}^{2}=0,
$$

which finishes the proof of Theorem 2.1 .

\section{ACKNOWLEDGEMENTS}

The authors thank Manoussos Grillakis and Sarada Rajeev for stimulating discussions during various stages of this project. They are also grateful to the referee for a number of helpful suggestions that led to improvements in the final version of this article. Both authors were supported in part by the National Science Foundation Career grant DMS-0747656. 


\section{REFERENCES}

1. P. Bizoń, T. Chmaj, and A. Rostworowski, Asymptotic stability of the skyrmion, Phys. Rev. D 75 (2007), no. 12, 121702-121706. MR2326835 (2008c:81110)

2. P. D'Ancona and V. Georgiev, Wave maps and ill-posedness of their Cauchy problem, New trends in the theory of hyperbolic equations, Oper. Theory Adv. Appl., vol. 159, Birkhäuser, Basel, 2005, pp. 1-111. MR2175916

3. D.-A. Geba, K. Nakanishi, and S. G. Rajeev, Global well-posedness and scattering for Skyrme wave maps, Commun. Pure Appl. Anal. 11 (2012), no. 5, 1923-1933. MR2911118

4. M. Gell-Mann and M. Lévy, The axial vector current in beta decay, Nuovo Cimento (10) 16 (1960), 705-726. MR0140316 (25:3738)

5. F. Gürsey, On the symmetries of strong and weak interactions, Nuovo Cimento (10) 16 (1960), 230-240. MR0118418(22:9191)

6. - On the structure and parity of weak interaction currents, Ann. Physics 12 (1961), no. 1, 91-117.

7. F. Hélein, Harmonic maps, conservation laws and moving frames, second ed., Cambridge Tracts in Mathematics, vol. 150, Cambridge University Press, Cambridge, 2002. Translated from the 1996 French original, with a foreword by James Eells. MR1913803 (2003g:58024)

8. J. Krieger, Global regularity and singularity development for wave maps, Surveys in differential geometry. Vol. XII. Geometric flows, Surv. Differ. Geom., vol. 12, Int. Press, Somerville, MA, 2008, pp. 167-201. MR2488946 (2010h:58044)

9. J. Krieger, W. Schlag, and D. Tataru, Renormalization and blow up for charge one equivariant critical wave maps, Invent. Math. 171 (2008), no. 3, 543-615. MR2372807 (2009b:58061)

10. A. N. Leznov, B. Piette, and W. J. Zakrzewski, On the integrability of pure Skyrme models in two dimensions, J. Math. Phys. 38 (1997), no. 6, 3007-3011. MR1449544 (98d:81047)

11. D. Li, Global wellposedness of hedgehog solutions for the $(3+1)$ Skyrme model, preprint, 2011.

12. F. Lin and Y. Yang, Existence of two-dimensional skyrmions via the concentrationcompactness method, Comm. Pure Appl. Math. 57 (2004), no. 10, 1332-1351. MR2070206 (2005d:58034)

13. Analysis on Faddeev knots and Skyrme solitons: recent progress and open problems, Perspectives in nonlinear partial differential equations, Contemp. Math., vol. 446, Amer. Math. Soc., Providence, RI, 2007, pp. 319-344. MR2376667 (2009c:58027)

14. P. Raphael and I. Rodnianski, Stable blow up dynamics for the critical co-rotational wave maps and equivariant Yang-Mills problems, Publ. Math. Inst. Hautes Études Sci. 115 (2012), 1-122. MR2929728

15. I. Rodnianski and J. Sterbenz, On the formation of singularities in the critical $\mathrm{O}(3) \sigma$-model, Ann. of Math. (2) $\mathbf{1 7 2}$ (2010), no. 1, 187-242. MR2680419 (2011i:58023)

16. J. Shatah, Weak solutions and development of singularities of the $\mathrm{SU}(2) \sigma-m o d e l$, Comm. Pure Appl. Math. 41 (1988), no. 4, 459-469. MR933231 (89f:58044)

17. J. Shatah and A. Tahvildar-Zadeh, Regularity of harmonic maps from the Minkowski space into rotationally symmetric manifolds, Comm. Pure Appl. Math. 45 (1992), no. 8, 947-971. MR:1168115 (93c:58056)

18. T. H. R. Skyrme, A non-linear field theory, Proc. Roy. Soc. London Ser. A 260 (1961), 127-138. MR0128862 (23:B1899)

19. _ Particle states of a quantized meson field, Proc. Roy. Soc. Ser. A 262 (1961), 237-245. MR0153323(27:3291)

20. A Anified field theory of mesons and baryons, Nuclear Phys. 31 (1962), 556-569. MR0138394 (25:1841)

21. D. Tataru, The wave maps equation, Bull. Amer. Math. Soc. (N.S.) 41 (2004), no. 2, 185-204. MR2043751 (2005h:35245)

Department of Mathematics, University of Rochester, Rochester, New York 14627

Department of Mathematics, University of Rochester, Rochester, New York 14627 